\title{
Evaluation of The Effectiveness of Alloplastic Prostheses for Atm Reconstruction: A Systematic Review
}

\author{
Thiago Raniel Nunes e Silva ${ }^{1 *}$, Giovana Ferreira Andrade ${ }^{1}$, Fabrício Ibiapina Tapety ${ }^{2}$, \\ José Zilton Lima Verde Santos ${ }^{3}$, Leonardo Alonso de Moura ${ }^{4}$, Tallita Barbosa Monteiro \\ dos Santos ${ }^{5}$, \\ ${ }^{1}$ Dental surgeon, Perfected in Minor Oral Surgery, Brazil \\ ${ }^{2}$ Dental surgeon from the Federal University of Piauí; Bauru as Doctorate in Oral Rehabilitation, Germany \\ ${ }^{3}$ Dental surgeon from the Federal University of Pernambuco; Master in Dentistry from the Federal University of Bahia, Brazil \\ ${ }^{4}$ Dental surgeon from the Faculty of Health, Human and Technological Sciences of Piauí, Brazil \\ ${ }^{5}$ Nutritionist graduated from the Federal University of Piauí, Brazil \\ *Corresponding author: Thiago Raniel Nunes e Silva, Dental surgeon, Perfected in Minor Oral Surgery, Brazil \\ Tallita Barbosa Monteiro dos Santos, Nutritionist graduated from the Federal University of Piauí, Brazil
}

\section{ARTICLE INFO}

Received: 慧 February 24, 2020

Published: March 09, 2020

Citation: Thiago R N e S, Giovana F A, Fabrício T, José Z L V S, Leonardo A de M, et al., Evaluation of The Effectiveness of Alloplastic Prostheses for Atm Reconstruction: A Systematic Review. Biomed J Sci \& Tech Res 26(3)-2020. BJSTR. MS.ID.004356.

Abbreviations: TMJ: Temporomandibular Joint; VAS: Visual Analogue Scale; AP: Alloplastic Prostheses

\section{ABSTRACT}

Objective: To evaluate the efficacy of total reconstruction of temporomandibular joint with alloplastic prostheses through a systematic literature review, focusing on pain, mouth opening and postoperative complications.

Method: This is a systematic review of the scientific production of the PubMed indexer. A combination of the terms "tmj reconstruction" and / or "temporomandibular joint prosthesis" and / or "tmj replacement" and / or "alloplastic prostheses" was used. We excluded studies with follow-up time of less than 2 years, studies published outside the current decade, case reports, animal studies and reviews. Clinical trials and prospective and retrospective observational studies that had preoperative and postoperative information on mouth openness, pain, incidence of complications and recurrence were included.

Results: We analyzed nine studies, all of them in the English. The mean follow-up ranged from 2 to 21 years. In total, 438 patients were evaluated, the majority being female. sssss

Conclusion: All articles presented positive data regarding improvements in pain and mouth opening in the postoperative period. The most frequent post-surgical complications were facial nerve injuries.

\section{RESUME}

Alloplastic TMJ prostheses are considered viable alternatives for the reconstruction of compromised Temporomandibular Joints and can bring important benefits to patients with advanced TMJ diseases. However, it has been observed that it is very difficult to predict many of the interactions between prosthesis and patient, and only with the results of clinical experiments and research is it possible to assess the success and failures in the use of these devices.

Goal: To evaluate the effectiveness of total reconstruction of the temporomandibular joint with alloplastic prostheses through a systematic literature review, focusing on the variables pain, mouth opening and post-surgical complications.

Method: This is a systematic bibliographic review of the scientific production of the PubMed index. A combination of the terms: "tmj reconstruction" and / or 
"temporomandibular joint prosthesis" and / or "tmj replacement" and / or "Alloplastic Prostheses (AP)" was used. Studies with a follow-up time of less than 2 years were excluded years, studies published outside the current decade, case reports, reports, animal studies and reviews. Clinical trials and prospective and retrospective observational studies that had pre- and postoperative information on mouth opening, pain, incidence of complications and recurrence were included.

Results: Nine (9) studies were analyzed, all in English. The average follow-up time ranged from 2 to 21 years. In total, 438 patients were evaluated, the vast majority of whom were female.

Conclusion: All articles brought positive data regarding improvements in pain and mouth opening Prostheses for TMJ are considered a viable option for the reconstruction of compromised temporomandibular joints, bringing important benefits to patients with advanced stage TMJ diseases. It has been observed, however, that it is very difficult to predict many of the interactions between prosthesis and patient, and only with results of clinical experiments and research it is possible to evaluate the success and the failures in the use of those devices.

\section{Introduction}

The reconstruction of the Temporomandibular Joint (TMJ) represents one of the most complex surgical approaches within maxillofacial surgery. The objectives of TMJ reconstruction include the three-dimensional restoration of joint morphology and function, reduction of existing symptoms and limitations, in addition to preventing the progression of some diseases [1]. Replacement of the TMJ with an alloplastic prosthesis is usually the last resort in cases of temporomandibular diseases in its terminal state [2]. The first alloplastic prosthesis for TMJ was developed in the 1960s by Christensen [3]. Since then, a range of new models and new techniques has been tested and improved, with the aim of promoting maximum use of these devices, bringing benefits to patients [4]. Alloplastic prostheses have been a widely accepted option and several surgeons have used them in different cases. The number of alloplastic prostheses implanted for reconstruction of mandibular segments involving the TMJ has grown significantly in recent decades, mainly due to the demand for quality of life of the population [5]. Some advantages of TMJ reconstructions with alloplastic materials include the possibility of starting physiotherapy in the immediate postoperative period, and the fact that a graft donor site is not necessary, which reduces surgical time and possible morbidity, resulting in fewer days inpatient [4] some complications can occur, such as fracture of the prosthesis, pain, infection, exposure and wear of the material, among others. This is because the location where the prosthesis is installed is extremely challenging, not only due to the biomechanical characteristics of the ATM, but also due to the remaining structures around it [6].

However, advances in imaging and advances in computing, including three-dimensional image processing with exact parameters, have allowed surgeons to more accurately diagnose their patients, in addition to enabling better planning and simulations, bringing ever more predictable results [7]. The ideal characteristics of a prosthesis include biocompatibility, functionality, low weight, adaptability, stability, resistance and nontoxic. These characteristics are the target of alloplastic material companies, in order to produce materials that can physiologically reconstruct the ATM and thus have increasingly predictable results [8]. However, it has been observed that it is very difficult to predict many of the interactions between prosthesis and patient, and only with the results of clinical experiences and research is it possible to evaluate the success and failures in the use of these devices, especially when talking about results in the medium and long term. Therefore, this study is of fundamental importance for decision making regarding the possibility of installing alloplastic prostheses in patients who need replacement or reconstruction of the TMJ, helping to systematically clarify some of the results provided by this type of device. The aim of this study is to evaluate the effectiveness of total reconstruction of the temporomandibular joint with customized and prefabricated alloplastic prostheses in solving pre-existing limitations and conditions through a systematic literature review, focusing on the variables pain, mouth opening and complications. post-surgical.

\section{Methodology}

This is a systematic bibliographic review study of the scientific production of the PubMed indexer about the effectiveness of using alloplastic prostheses for the reconstruction of the temporomandibular joint, focusing on the variables mouth opening, pain and complications. A combination of the following search terms was used: "TMJ reconstruction" and / or "temporomandibular joint prosthesis" and / or "TMJ replacement" and / or "alloplastic prostheses". The results were submitted to the classification and inclusion protocols of the studies.

Controlled clinical trials and prospective and retrospective observational studies in humans, with more than 10 cases studied, comparing or containing results of surgical techniques for TMJ 
reconstruction and which had pre and postoperative information on mouth opening, pain and incidence complications were included. Studies with a follow-up period of less than 2 years (average), studies published outside the current decade (before 2010, last 7 years) were excluded. Case reports, technical reports, animal studies and reviews were excluded. The direct result of the combined search resulted in 1992 articles that were submitted to automatic indexer filters (year of publication and language). 442 studies were selected from the database after the application of automatic indexer filters and from the thorough reading of the titles of the 442 studies, and based on the evaluators' interpretation, 34 studies were considered potentially useful. These 34 studies were read in full in search of the necessary data for the execution of this research (data related to follow-up time, pain, mouth opening and complications), and disagreements related to the selected studies were resolved through a discussion based on the relevance of the study to the work, including missing data, form of data presentation and other criteria mentioned above. The final sample consisted of 9 studies from which the following data were extracted: authors, study design, year of publication, number of patients, gender (exact proportion or number), age of the patients (mean or range), measure of pre and postoperative mouth opening (in $\mathrm{mm}$ ), presence of pre and postoperative pain (Visual Analogue Scale - VAS), and follow-up period (average or fixed). The graph below (Figure 1) illustrates the systematic of this research.

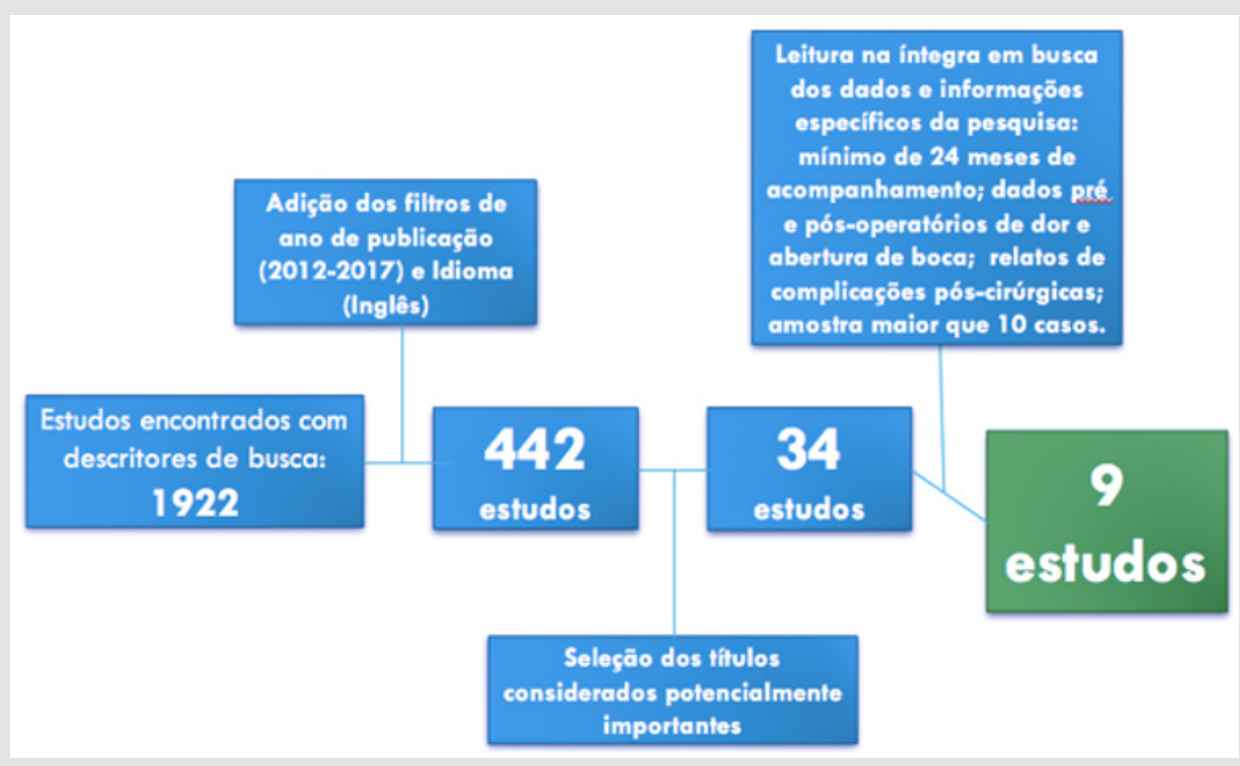

Figure 1: Flowchart of the systematic study selection process.

Table 1: Characteristics of the included studies.

\begin{tabular}{|c|c|c|c|c|c|c|}
\hline Authors & Type & Year & Patients & Genre & Age & Acomp \\
\hline Westermark [11] & Prospective & 2010 & 12 & $09 \mathrm{~F}-3 \mathrm{M}$ & $14-53$ & 8 years \\
\hline Machon et al. [10] & Prospective & 2011 & 27 & $21 \mathrm{~F}-6 \mathrm{M}$ & $18-60$ & 2 years \\
\hline $\begin{array}{l}\text { Aagaard e } \\
\text { Thygesen }\end{array}$ & Prospective & 2014 & 61 & Not reported & $16-41$ & 3 years \\
\hline Burgess et al. [13] & Retrospective & 2014 & 52 & $44 \mathrm{~F}-8 \mathrm{M}$ & $20-84$ & 3 years \\
\hline $\begin{array}{l}\text { Gruber [12], } \\
\text { McCullough e } \\
\text { Sidebottom. }\end{array}$ & Prospective & 2015 & 84 & $75 F-9 M$ & $19-72$ & 5 years \\
\hline Sanovich et al. [14] & Retrospective & 2014 & 62 & $62 \mathrm{~F}-0 \mathrm{M}$ & Dec-50 & $\begin{array}{r}\text { Up until } \\
7 \text { years }\end{array}$ \\
\hline $\begin{array}{l}\text { Gonzalez-Perez } \\
\text { et al. }\end{array}$ & Prospective & 2015 & 52 & $35 \mathrm{~F}-17 \mathrm{M}$ & $29-74$ & $\begin{array}{c}2 \\
\text { years }\end{array}$ \\
\hline Wolford et al. [8] & Prospective & 2015 & 56 & $52 \mathrm{~F}-04 \mathrm{M}$ & 38.6 (média) & $\begin{array}{c}21 \\
\text { years }\end{array}$ \\
\hline
\end{tabular}




\begin{tabular}{|r|r|r|c|c|c|c|}
\hline \multirow{2}{*}{ Wolford et al. [8] } & Retrospective & 2016 & 32 & $22 \mathrm{~F}-10 \mathrm{M}$ & $11-68$ & $\begin{array}{c}\text { Up until } \\
14 \\
\text { years }\end{array}$ \\
\hline
\end{tabular}

Table 2: Description of pre and postoperative results of mouth opening and pain, and complications.

\begin{tabular}{|c|c|c|c|c|c|}
\hline \multirow[t]{2}{*}{ Authors } & \multicolumn{2}{|c|}{ Mouth opening (Average in millimeters) } & \multicolumn{2}{|c|}{ Pain (EVA [0-10]) } & \multirow{2}{*}{$\begin{array}{c}\text { Complications } \\
\text { (By event) }\end{array}$} \\
\hline & Pre-OP & Pós-OP & Pré-OP & Pós-OP & \\
\hline Westermark [11] & 16,41 & 33,16 & 9-Jul & 0 & $\begin{array}{l}1 \text { lymphadenopathy; } \\
1 \text { buzz }\end{array}$ \\
\hline Machon et al. [10] & 17.7 & 29.1 & $4(0-5)^{*}$ & $2.1(0-5)^{*}$ & $\begin{array}{l}9 \text { paresthesia of the } \\
\text { facial nerve; } 2 \text { injury } \\
\text { to the mental nerve; } 4 \\
\text { muscle pain; } 5 \text { mental } \\
\text { disorders; } 4 \text { occlusal } \\
\text { instability. }\end{array}$ \\
\hline Aagaard e Thygesen & 29.5 & 31.8 & 7.2 & 1.6 & $\begin{array}{l}1 \text { persistent edema; } \\
1 \text { allergy to Cr-Co; } 2 \\
\text { surgical revisions }\end{array}$ \\
\hline \multirow[t]{2}{*}{ Burgess et al. [13] } & - & - & & & $\begin{array}{c}3 \text { neuropathy; } 1 \\
\text { paraesthesia; } 10 \\
\text { paraesthesia; } 7 \text { facial } \\
\text { nerve injury; } 1 \text { Frey } \\
\text { syndrome; } 1 \text { cheeloid; } \\
1 \text { limited mouth } \\
\text { opening }\end{array}$ \\
\hline & & & $\begin{array}{l}\text { Customized } 5.8^{* *} \\
\text { Stock 5.4** }\end{array}$ & & \\
\hline $\begin{array}{l}\text { Gruber [12], } \\
\text { McCullough e } \\
\text { Sidebottom }\end{array}$ & 21 & 33.7 & 7.4 & 0.8 & $\begin{array}{l}1 \text { infection; } 1 \text { facial } \\
\text { cellulite -> removal; } \\
1 \text { paresthesia of } \\
\text { the facial nerve; } 2 \\
\text { displacements }\end{array}$ \\
\hline Sanovich et al. [14] & 26.1 & 34.4 & 7.9 & 3.8 & $\begin{array}{l}2 \text { heterotopic bone -> } \\
\text { removal; } 1 \text { infection } \\
\text {-> removal; } 1 \text { screw } \\
\text { failure -> removal }\end{array}$ \\
\hline Gonzalez-Perez et al. & 27.1 & 42 & 6.4 & 1.6 & $\begin{array}{l}3 \text { malocclusions; } 2 \\
\text { temporary paresthesia } \\
\text { of the facial; } 1 \text { cobalt } \\
\text { / chromium allergy -> } \\
\text { replacement; } 1 \text { open } \\
\text { bite -> re-approach; } 1 \\
\text { class II malocclusion -> } \\
\text { re-approach }\end{array}$ \\
\hline Wolford et al. [8] & 25.8 & 36.2 & 8 & 3 & Not reported \\
\hline Wolford et al. [8] & 14.5 & 35 & 8 & 1.5 & $\begin{array}{c}6 \text { re-approach; } 2 \\
\text { heterotopic bone -> re- } \\
\text { approach; } 1 \text { infection; } \\
1 \text { SAPHO syndrome -> } \\
\text { removal }\end{array}$ \\
\hline
\end{tabular}

\section{Results}

A total of 9 (nine) studies were included, all in English, of which 6 (six) were prospective cohort studies and 3 (three) were retrospective studies. Detailed data for the nine studies are shown in Table 1 . The average follow-up time ranged from 2 to 21 years. In total, 438 patients were evaluated, the vast majority of whom were female (gender data missing in 1 of 9 articles). TMJ surgical approaches were the same in all studies that reported them. 
Six of the nine articles reported the technique used to install the prostheses, which consisted of pre-auricular access combined with submandibular or retromandibular access. Three articles did not report the technique used. Data regarding mouth opening, pre- and postoperative pain and complications are available in Table 2.

\section{Discussion}

This review study sought, in a systematic way, to evaluate the efficacy of TMJ alloplastic prostheses for the treatment of diseases thataffect this joint, evaluating the variables pain, mouth opening and complications. The selected and studied articles are heterogeneous as to the type of study, sample size, materials, systems and patient characteristics, therefore, the present results should be interpreted with caution. Regarding pain, the data present in one of the studies [9] showed subjective pain relief for up to 3 years after surgery in most patients and in the global average (Table 2), although some patients have persistently experienced postoperative pain levels, or even greater than pre-surgical. The author does not specify the number of individuals, nor the indication for TMJ reconstruction for these patients, but states that some would need psychological therapy after surgery, inquiring about the possible psychological factor in maintaining chronic pain even after surgical interventions completed with a certain level of success. A similar fact occurs in another study [10], where the subjective pain report was reduced in 15 patients (53\% of the total studied), from an average of 4 (range $0-5$ on the EVA scale) preoperatively to an average of 2, 1 in the postoperative period.

However, 4 patients reported worsening postoperative pain. The results of another publication [7] also showed a significant reduction in pain intensity after two years of follow-up. There was a significant reduction in pain in 25 cases of TMJ replacement (48\% of the patients studied), with a reduction in pain of more than $80 \%$ (compared to preoperative pain values). Twelve of these 25 patients ( $23 \%$ of the total cases) were in the $100 \%$ pain reduction category, that is, with a postoperative pain score of 0 (total absence of pain). The other studies reported pain reduction based on the global average of their results [8,10-12]. Regarding mouth opening, all studies have shown positive results with regard to improvement in mouth opening [7-15]. However, one of the studies 13 reports that of the 12 patients in the sample, two had no gains in mouth opening. These two patients admitted that they did not perform the mandible opening exercises, required by the preoperative agreement, which indicates that the postoperative physical exercises are important so that the patients reached an acceptable opening capacity.

The results of another study [7] show that there was an improvement of mouth opening 2 years after surgery (Table 2), although the author himself acknowledged that there was a discrepancy in the distribution of data, since some patients gain), which makes the distribution highly asymmetric, tilting to the right. A similar situation is also identified in another publication
[12], where the specific group of ankylosing patients had much greater improvements in mouth opening compared to the rest of the sample, which also generates a bias in the study result, since ankylosing patients have marked signs disease, limitation of mouth opening. The complications reported in the studies were quite standardized, with facial nerve injuries being the most common [7,10-12]. One of the studies [11] points out that $27 \%$ of patients reported adverse problems even after 1 year of surgery (Table 2 ), a relatively high rate of complications. However, for the group with persistent complications, the average number of previous procedures was 2.60 compared to an average of 1.77 for the group without complications. This finding suggests that the number of previous operations is a significant predictor of adverse outcomes in the postoperative period. One study did not report postoperative complications.

\section{Conclusions}

TMJ reconstructions with alloplastic materials reduce the perception of pain after the surgical procedure of installation, in addition to improvements in the opening of the mouth that are maintained even for long periods of follow-up. The most frequent complications were injuries to the facial nerve and its branches.

\section{References}

1. Mercuri LG (2013) The role of custom-made prosthesis for temporomandibular joint replacement. Revista Espanhola de Cirurgia Oral e Maxilofacial 35(1): 1-10.

2. Johnson NR, Roberts MJ, Doi SA, Batstone MD (2016) Total temporomandibular joint replacement prostheses: a systematic review and bias- adjusted meta-analysis. Journal of Oral and Maxillofacial Surgeons. 46(1): 86-92.

3. Shan Yong Z, Liu H, Yang C, Zhang X, Abdel Rehem A, et al. (2015) Modified surgical techniques for total alloplastic temporomandibular joint replacement: One institution's experience. Journal of CranioMaxillo-Facial Surgery 43(6): 934-939.

4. Ferreira FM, Cunali RS, Bonotto D, Farias ACd, Cunali PA (2014) Total temporomandibular joint alloplastic reconstruction. Revista Dor de São Paulo 15 (3): 211-214.

5. Guarda Nardini L, Manfredini D, Ferrovato G (2008) Total temporomandibular joint replacement: A clinical case with a proposal for post-surgical rehabilitation. Journal of Cranio-Maxillofacial Surgery 36(7): 403-409.

6. Marx RE, Cillo JE, Broumand V, Ulloa JJ (2008) Outcome Analysis of Mandibular Condylar Replacements in Tumor and Trauma Reconstruction: A Prospective Analysis of 131 Cases with Long-Term Follow-Up. Journal of Oral Maxillofacial Surgery 66(12): 2515-2523.

7. Gonzalez Perez LM, Fakih Gomez N, Gonzalez Perez Somarriba, Centeno G, Montes Carmona F (2016) Two-year prospective study of outcomes following total temporomandibular joint replacement. International Journal of Oral and Maxillofacial Surgery 45(1): 78-84.

8. Wolford L, Movahed R, Teschke M, Fimmers R, Havard D (2016) Temporomandibular Joint Ankylosis Can Be Successfully Treated with TMJ Concepts Patient-Fitted Total Joint Prosthesis and Autogenous Fat Grafts. Journal of Oral Maxillofacial Surgery 74(6): 1215-1227.

9. Aagaard E, Thygesen T (2014) A prospective, single-center study on patient outcomes following temporomandibular joint replacement using a custom-made Biomet TMJ prosthesis. International Journal of Oral and Maxillofacial Surgery 43(10): 1229-1235. 
10. Machon V, Hirjak D, Beno M, Foltan R (2012) Total alloplastic temporomandibular joint replacement: the Czech-Slovak initial experience. International Journal of Oral and Maxillofacial Surgery 41(4): 514-517.

11. Westermark A (2010) Otal reconstruction of the temporomandibular joint. Up to 8 years of follow-up of patients treated with Biomet tota joint prostheses. International Journal of Oral Maxillofacial Surgery 39(10): 951-944.

12. Gruber EA, McCullough J, Sidebottom AJ (2015) Medium-term outcomes and complications after total replacement of the temporomandibular joint. Prospective outcome analysis after 3 and 5 years. British Journal of Oral and Maxillofacial Surgery 53(5): 412-415.

\section{ISSN: 2574-1241}

DOI: $10.26717 /$ BJSTR.2020.26.004356

Thiago Raniel Nunes e Silva. Biomed J Sci \& Tech Res

(C) This work is licensed under Creative

Submission Link: https://biomedres.us/submit-manuscript.php
13. Burgess M, Bowler M, Jones R, Hase M (2015) Improved Outcomes After Alloplastic TMJ Replacement: Analysis of a Multicenter Study from Australia and New Zealand. Journal of Oral and Maxillofacial Surgery 72(7): 1251-1257.

14. Sanovich R, Mehta U, S Abramowicz, Widmer C, F Dolwick M (2014) Total alloplastic temporomandibular joint reconstruction using Biomet stock prostheses: The University of Florida experience. International Journal of Oral and Maxillofacial Surgery 43(9): 1091-1095.

15. Wolford LM, Mercuri LG, Schneiderman ED, Movahed R, Allen W (2015) Twenty-Year Follow-up Study on a Patient-Fitted Temporomandibular Joint Prosthesis: The Techmedica/TMJ Concepts Device. Journal of Oral and Maxillofacial Surgery 73(5): 952-960.

$\begin{array}{ll}\text { BIOMEDICAL } & \text { Assets of Publishing with us } \\ \text { RESEARCHES } & \text { - Global archiving of articles } \\ & \text { - Immediate, unrestricted online access } \\ & \text { - Rigorous Peer Review Process } \\ \end{array}$

